\title{
Bilateral Nevus Comedonicus Successfully Treated with Topical Adapalene: A Case Report
}

\section{Mahran AM*, Abdelsamea GM and Mekkawy MM \\ Department of Dermatology, Venereology and Andrology; Assiut University, Egypt}

*Corresponding author: Ayman M Mahran, MD, Department of Dermatology, Venereology and Andrology; Faculty of Medicine, Assiut University, Assiut; Egypt, Tel: 00201211000033; E-mail: aymanderma@yahoo.com

\section{Case Report \\ Volume 2 Issue 4}

Received Date: November 04, 2017

Published Date: November 13, 2017

DOI: $10.23880 /$ cdoaj- 16000136

\begin{abstract}
Nevus comedonicus (NC) is a rare type of epidermal nevi characterized by aggregation of dilated follicular orifices filled with keratin. Although most cases occur unilaterally on the face, rarely, it may show a bilateral distribution (bilateral nevus comedonicus). Here, we report an unusual case of bilateral nevus comedonicus in a 7-year-old female child without systemic associations. In addition, and for the first time to be reported, she was treated successfully with Adapalene gel $0.1 \%$.
\end{abstract}

Keywords: Nevus comedonicus; Nevus Comedonicus Syndrome; Adapalene

Abbreviations: NC: Nevus Comedonicus; NEK9: NIMA-related kinase9; RAR: Retinoic acid Receptor.

\section{Introduction}

Nevus comedonicus is composed of close, dilated follicular openings with keratin plugs resembling comedones. It is a rare type of epidermal nevi with a predilection for face and neck [1]. Although the majority of cases occur unilaterally; NC rarely show a bilateral distribution [2]. Most cases are isolated; however, it may be a part of nevus comedonicus syndrome [3].

Prevalence of NC ranges from 1 in 45,000 to 1 in 100,000 with no gender or racial preference [1]. Approximately, $50 \%$ of cases of NC are evident at birth. However, delayed development was reported [3]. As NC is benign, treatment is indicated only for cosmetic concerns [4].

\section{Case Report}

A 7-year-old Upper Egyptian girl was referred to our outpatients' clinic, Assiut University Hospitals, Assiut, Egypt, complaining of bilateral and symmetrical skin lesions on both cheeks of 3 years duration. Lesions gradually increased in size and number with time. The patient received various topical treatments with no satisfactory improvement. Lesions were asymptomatic. Medical history was unremarkable with no positive family history. No positive consanguinity between parents. Pregnancy and labor were uneventful. Mother denied any drug intake during pregnancy.

Ophthalmological, neurological, abdominal, cardiac and chest examinations did not reveal any abnormality. Her vital signs, body weight, length and head circumference were normal for age. Laboratory tests (CBC, blood sugar, lipogram, liver functions and kidney functions) were unremarkable. 
Local examination revealed two slightly hyperpigmented plaques, each formed of closely set papules studded with black comedo-like lesions. The lesion on the right cheek measured $7 \times 2.5 \mathrm{~cm}$ and the one on the left measured $6 \times 3 \mathrm{~cm}$ (Figures 1-3). Mild dryness with exfoliation was observed. No other cutaneous abnormalities could be detected. Also, hair and nails as well as mucous membranes were clinically normal. A punch biopsy was obtained from the right cheek. Histopathologic examination revealed dilated and invaginated follicular structures filled with lamellar keratin and devoid of hair shafts. Epidermis was almost normal (Figure 4). Based on clinicopathological correlation, the diagnosis of bilateral nevus comedonicus was made. Patient was treated with topical Adapalene gel $0.1 \%$ once daily. She was regularly followed up and after 4 months, she showed excellent improvement, in terms of the pigmentation and disappearance of the comedo-like lesions without any side effect (Figures 1-3).

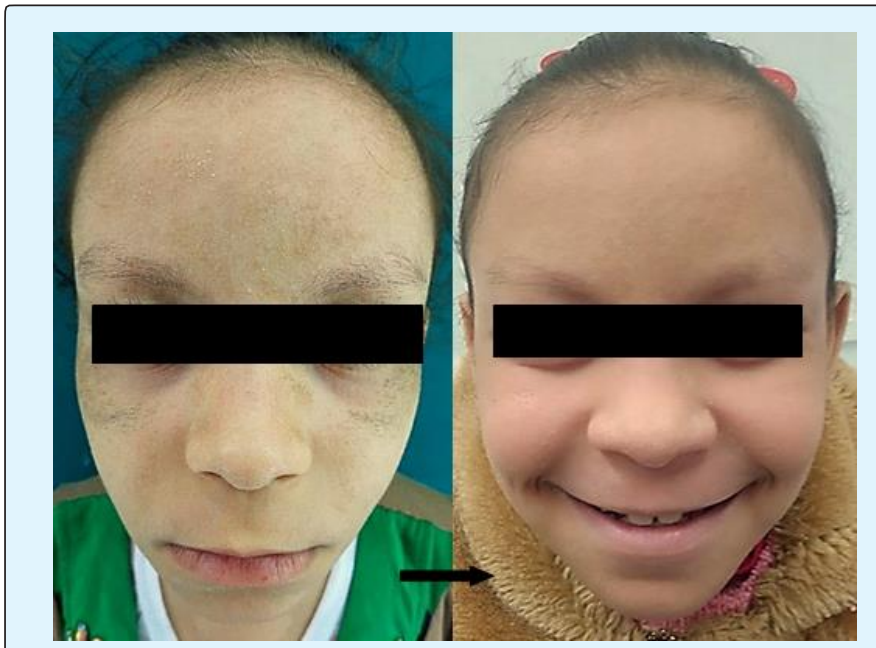

Figure 1: Clinical features before and after treatment, front view.

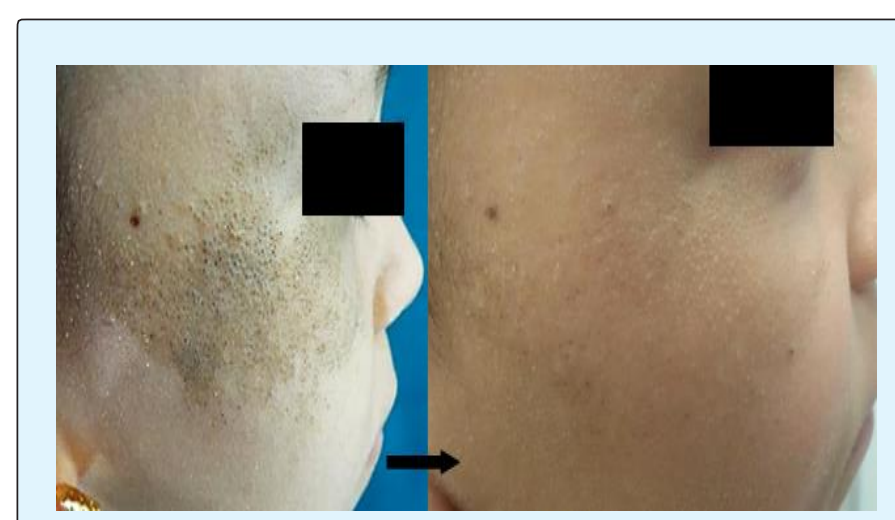

Figure 2: Clinical features before and after treatment, right lateral view.

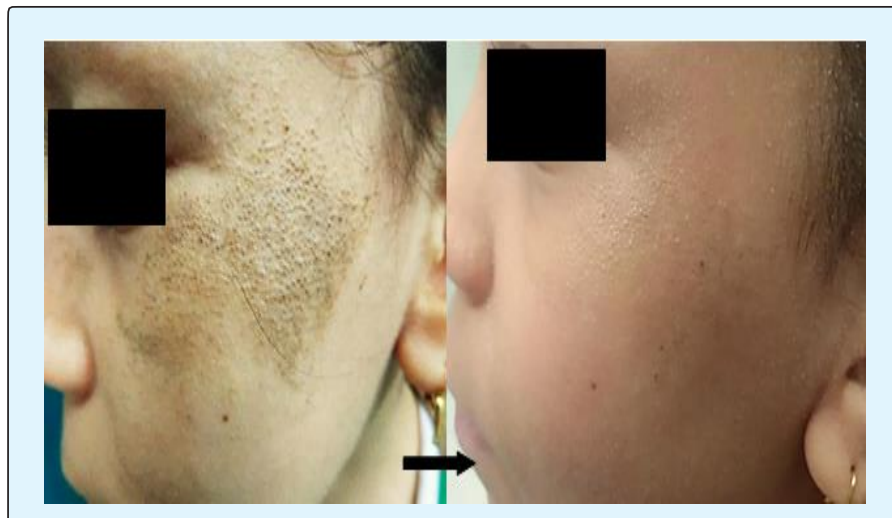

Figure 3: Clinical features before and after treatment, left lateral view.

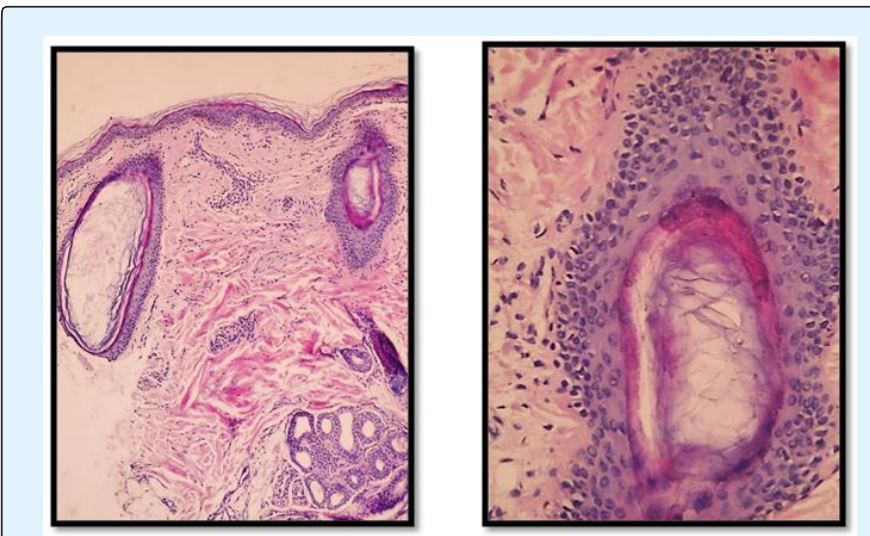

Figure 4: Microscopic features by Hematoxylin-Eosin staining with magnification power $(\mathrm{x} 100)$ for the left photo and $(\mathrm{x} 400)$ on the right photo.

\section{Discussion}

The exact etiopathogenesis of NC is unclear. Many consider it an adnexal hamartoma, which forms as a consequence of a developmental failure of the mesodermal component of the pilosebaceous unit, resulting in abnormal differentiation of the epithelial component [5]. The abnormal follicular structure becomes unable to form terminal hair, sebaceous glands or arrector pili muscle and becomes capable only of producing keratin. Keratin accumulates in the adnexal orifices, producing its characteristic comedo-like appearance [1,5]. Others consider it a nevus of hair follicle or sweat ducts [6]. On genetic basis, it was found that somatic mutations of NEK9 (NIMA-related kinase9) are responsible for the abnormal follicular differentiation [7].

Clinically, NC appears as a collection of discrete, dilated follicular ostia plugged with pigmented keratin. Usually it 


\section{Clinical Dermatology Open Access Journal}

is unilateral but, rarely, can be bilateral [8]. Sometimes, secondary inflammatory changes are seen with significant scarring [2].

The reported therapeutic responses of nevus comedonicus to various topical and systemic therapeutics were insufficient and unsatisfactory. To the best of our knowledge, there are no published data about the effects of Adapalene as a possible therapeutic option for NC and our report is the first to highlight its successful use. Adapalene is a third generation synthetic retinoid, which has high affinity to retinoic acid receptor (RAR) $\beta$ and $\gamma$ unlike tretinoin. This is important because epithelial cells mainly have RAR $\gamma$ [9]. It inhibits micro-comedons formation and decreases keratinocytes' cohesiveness in sebaceous follicles. It has an anti-inflammatory effect which is due to suppression of the lipooxygenase activity and due to the oxidative metabolism of arachidonic acid. This effect might be the reason for decreased irritation with Adapalene. Common side effects of Adapalene include erythema, peeling, dryness and burning [10].

Differential diagnosis of our case revolves around familial dyskeratotic comedones, idiopathic disseminated comedones, multiple congenital comedones and acne vulgaris. However, the characteristic clinicopathological features of the aforementioned differential diagnoses were enough to exclude them from the diagnosis of our case. So, based on the clinicopathological features, the diagnosis of bilateral NC was made for our case.

In conclusion, we report for the first time in the literature a rare case of bilateral $\mathrm{NC}$ who was successfully treated by once daily Adapalene gel $0.1 \%$ for a period of 4 months without any obvious side effect. We recommend the need for further studies to validate the use of Adapalene as an effective and safe therapeutic modality for this cosmetically annoying disorder.

\section{References}

1. Nabai H, Mehregan AH (1973) Nevus comedonicus. A review of the literature and report of twelve cases. Acta Derm Venereol 53(1): 71-74.

2. Paige TN, Mendelson CG (1967) Bilateral nevus comedonicus. Arch Dermatol 96(2): 172-175.

3. Patrizi A, Neri I, Fiorentini C, Marzaduri S (1998) Nevus comedonicus syndrome: a new pediatric case. Pediatr Dermatol 15(4): 304-306.

4. Tchernev G, Ananiev J, Semkova K, Dourmishev LA, Schönlebe J (2013) Nevus Comedonicus: An Updated Review. Dermatol Ther 3(1): 33-40.

5. Wood MG, Thew MA (1968) Nevus comedonicus. A case with palmar involvement and review of the literature. Arch Dermatol 98(2): 111-116.

6. Lefkowitz A, Schwart RA, Lambert WC (1999) Nevus comedonicus. Dermatology 199:204-207.

7. Levinsohn JL, Sugarman JL, McNiff JM, Antaya R, Choate KA (2016) Somatic Mutations in NEK9 Cause Nevus Comedonicus. Am J Hum Genet 98(5): 10301037.

8. Sikorski D, Parker J, Shwayder T (2011) A boy with an unusual scalp birthmark. Nevus Comedonicus. Int J Dermatol 50(6): 670-672.

9. Czernielewski J, Michel S, Bouclier M, Baker M, Hensby JC (2001) Adapalene biochemistry and the evaluation of a new topical retinoid for treatment of acne. J Eur Acad Dermatol Venereo 15(3): 5-12.

10. Millikan LE (2000) Adapalene: an update on newer comparative studies between the various retinoids. Int J Dermatol 39(10): 784-788. 